pp 1301-1317. (C) The Author(s), 2020. Published by Cambridge University Press on behalf of Royal Aeronautical Society. This is an Open Access article, distributed under the terms of the Creative Commons Attribution licence (http://creativecommons.org/licenses/by/4.0/), which permits unrestricted re-use, distribution, and reproduction in any medium, provided the original work is properly cited.

doi:10.1017/aer.2020.44

\title{
CFD study of oil-jet gear interaction flow phenomena in spur gears
}

\author{
M.C. KelleriD, C. Kromer, L. Cordes, C. Schwitzke and H.-J. Bauer \\ marc.keller@kit.edu \\ Institute of Thermal Turbomachinery (ITS) \\ Karlsruhe Institute of Technology (KIT) \\ Karlsruhe \\ Germany
}

\section{ABSTRACT}

Oil-jet lubrication and cooling of high-speed gears is frequently employed in aeronautical systems, such as novel high-bypass civil aero engines based on the geared turbofan technology. Using such oil-jet system, practitioners aim to achieve high cooling rates on the flanks of the highly thermally loaded gears with minimum oil usage. Thus, for an optimal design, detailed knowledge about the flow processes is desired. These involve the oil exiting the nozzle, the oil impacting on the gear teeth, the oil spreading on the flanks, the subsequent oil fling-off, as well as the effect of the design parameters on the oil flow. Better understanding of these processes will improve the nozzle design phase, e.g. regarding the nozzle positioning and orientation, as well as the nozzle sizing and operation.

Most related studies focus on the impingement depth to characterize the two-phase flow. However, the level of information of this scalar value is rather low for a complete description of the highly dynamic three-dimensional flow. Motivated by the advancements in numerical methods and the computational resources available nowadays, the investigation of the oil-jet gear interaction by means of computational fluid dynamics (CFD) has come into focus lately.

In this work, a numerical setup based on the volume-of-fluid method is presented and employed to investigate the two-phase flow phenomena occurring in the vicinity of the gear teeth. The setup consists of a single oil-jet impinging on a single rotating spur gear. By introducing new metrics for characterizing the flow phenomena, extensive use of the possibilities of modern CFD is made, allowing a detailed transient and spatially resolved flow analysis. Thus, not only the impingement depth, but also the temporal and spatial evolution of wetted areas on the gear flanks, as well as the evolution of the oil volume in contact with the gear flanks are extracted from the simulation data and compared in a CFD study.

The study consists of 21 different simulation cases, whereby the effect of varying the jet velocity, the jet inclination angle, the jet diameter, and the gear speed are examined. Consistent results compared to a simplified analytical approach for the impinging depth are obtained and the results for the newly introduced metrics are presented.

Keywords: Gear lubrication and cooling; Two-phase flow; Volume-of-fluid (VOF) method 


\section{NOMENCLATURE}

$\begin{array}{ll}\text { AMR } & \text { adaptive mesh refinement } \\ \text { BC } & \text { boundary condition } \\ \text { CFD } & \text { computational fluid dynamics } \\ \text { OGI } & \text { oil-jet gear interaction } \\ \text { SPH } & \text { Smoothed Particle Hydrodynamics } \\ \text { VOF } & \text { Volume-of-Fluid }\end{array}$

\section{Latin Symbol}

$\begin{array}{ll}A_{\mathrm{f}} & \text { gear flank surface area } \\ A_{\mathrm{w}} & \text { wetted area } \\ C & \text { volume fraction } \\ d_{\text {jet }} & \text { jet diameter } \\ d_{\mathrm{n}} & \text { nozzle bore hole diameter } \\ D_{\mathrm{i}} & \text { impingement depth } \\ D_{\mathrm{p}} & \text { penetration depth } \\ H & \text { gear tooth height } \\ l_{\mathrm{n}} & \text { nozzle channel length } \\ m & \text { gear module } \\ N & \text { no. of gear teeth } \\ Q & \text { volumetric oil flow rate } \\ R & \text { gear pitch radius } \\ R_{\text {interface }} & \text { radial interface position } \\ R_{\mathrm{n}} & \text { nozzle outlet radius } \\ R_{\text {top }} & \text { radial confinement radius } \\ S & \text { contour coordinate along gear tooth profile } \\ t & \text { time } \\ v_{\text {jet }} & \text { jet velocity } \\ v_{\mathrm{p}} & \text { pitch line velocity } \\ V_{\text {gap }} & \text { fed oil volume per gear tooth gap } \\ V_{\mathrm{w}} & \text { oil film volume } \\ w & \text { domain width } \\ x_{\mathrm{a}} & \text { gear addendum modification coefficient } \\ & \end{array}$

\section{Greek symbol}

$\begin{array}{ll}\alpha_{\text {jet }} & \text { jet inclination angle } \\ \hat{\alpha}_{\text {jet }} & \text { effective jet inclination angle } \\ \gamma & \text { fluid phase } \\ \theta & \text { contact angle }\end{array}$




$\begin{array}{ll}\mu & \text { dynamic viscosity } \\ \rho & \text { density } \\ \sigma & \text { surface tension coefficient } \\ \Sigma & \text { jet to gear pitch line velocity ratio } \\ \phi & \text { fluid property } \\ \omega & \text { gear speed }\end{array}$

\subsection{INTRODUCTION}

Better understanding of the oil-jet gear interaction (OGI) is vital for a robust and efficient design of reduction units for geared turbofan technology. Since overall engine efficiency targets, such as minimal weight or power losses, require minimum use of oil, an optimal design of the cooling and lubrication system is desired. The high-speed gears are usually lubricated and cooled by an oil-jet system in which oil is jetted directly onto the gear flanks resulting in the complex flow pattern of the OGI, illustrated in Fig. 1.

The supplied oil is guided through a nozzle and injected in the vicinity of the gear teeth. For the radially oriented jet at the top, the subsequent flow phases are schematically shown. The oil-jet impacts on the gear flank, which is associated with a strong deformation of the jet tip. Then, the oil on the gear flank forms an oil film that spreads towards the bottom land but also in the axial direction. Consequently, the oil is flung off by centrifugal forces. Fondelli et al. ${ }^{(1)}$ and Keller et al. ${ }^{(2)}$ present characteristic non-dimensional groups. They show that while the impingement and spreading is mainly driven by inertia forces, the film motion is driven by centrifugal, viscous, and to a lesser extent by surface tension forces.

In Fig. 1, inclined oil-jet nozzles are schematically included for angles $\alpha_{\text {jet }}<0$ against the direction of rotation of the gear (left) and angles $\alpha_{\text {jet }}>0$ in the direction of rotation (right). It is obvious that in these cases the initial oil impact and, thus, the whole OGI will behave differently due to the changed kinematics. Neglecting variations on the gear design, further dominant parameters that affect the OGI are: the jet velocity $v_{\text {jet }}$, the jet diameter $d_{\text {jet }}$, the gear speed $\omega$, and material properties. The diversity of independent variables quickly leads to a wide parameter space, which is why the complete description of the system is challenging.

In literature, the impingement depth ${ }^{(3,4,5)}$ is frequently used as a basic design parameter. However, the value only provides information about how far the jet tip reaches into the tooth gap but does not contain information about the three-dimensional spreading behavior. Since this is crucial knowledge for an accurate modeling of the thermal behavior ${ }^{(6,7)}$ and also for a reliable fulfillment of the lubrication requirements ${ }^{(3)}$, there is still the need for a more detailed understanding and description of the oil flow after the oil-jet impact.

Up until now, only few numerical investigations have addressed the topic and experiments $^{(8,4,9,10)}$ face limitations due to a challenging experimental access and high demands on measurement equipment. However, through the increased availability of computational resources, the numerical simulation of the OGI became feasible in the last decade. Yazdani and Soteriou ${ }^{(11)}$ presented a method to investigate the long-term thermal behavior of gears by computational fluid dynamics (CFD). The OGI was simulated by means of the Volume-of-Fluid (VOF) method for two directions of rotation of a single gear. The authors highlighted the large dependency of oil flow patterns on mesh resolution by comparing results with and without adaptive mesh refinement (AMR). Although they mentioned the importance 


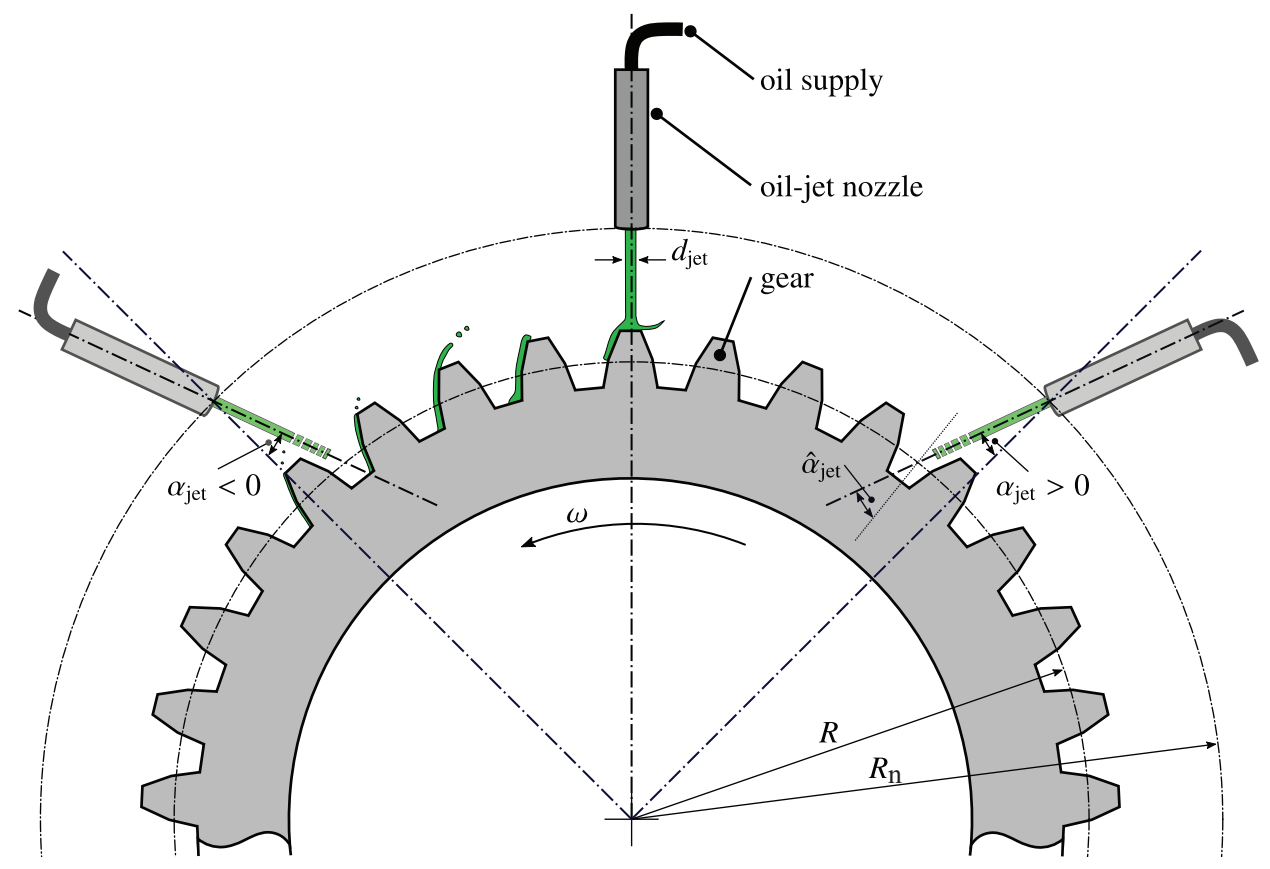

Figure 1. Schematic illustration of gear lubrication and cooling by means of oil-jets.

of an accurate modeling of the oil distribution near the gear surface, the small-scale flow phenomena were not captured in detail. Fondelli et al. ${ }^{(12)}$ conducted more fine-resolved CFD simulations. They presented a CFD setup, also using the VOF method but with a more detailed focus on the OGI itself. The goal of the investigations was to analyse the resistant torque and its link to the OGI. A sensitivity study illustrated the strong influence of mesh resolution and different AMR strategies on the simulation results. In a further work by the authors ${ }^{(1)}$, the isothermal setup was used to investigate the influence of the oil-jet inclination angle on the resistant torque. A challenging aspect about the OGI simulation is the incorporation of the gear rotation and gear meshing. Therefore, alternative CFD approaches have been employed. Keller et al. ${ }^{(13,2)}$ applied the particle-based Smoothed Particle Hydrodynamics (SPH) method to simulate the interaction between an oil-jet and a rotating gear. In a comparison to results obtained using the VOF method, consistent flow patterns were observed, substantiating the validity of the numerical predictions. The resulting flow patterns following the jet impact for three different jet inclination angles were examined, yielding qualitative insights into the OGI. The simulated cases were recently adopted by Ambrose et al. ${ }^{(14)}$, who employed the Lattice Boltzmann method and found comparable qualitative results.

Further CFD studies exist which focus on the effect of oil-jet lubrication on the global load-independent power losses ${ }^{(15,16,17)}$. While consistent torque results to analytical models were found in these investigations, the results are not used to quantitatively characterise the oil flow phenomena.

The goal of the present paper is to investigate the OGI between a single oil-jet and a single rotating gear by means of the VOF method and to make use of an extended post-processing 


\section{Table 1}

Material properties of simulated fluids

\begin{tabular}{lccc} 
Property & Unit & Air & Oil \\
Density $\rho$ & $\mathrm{kg} / \mathrm{m}^{3}$ & 0.9862 & 949 \\
Dynamic viscosity $\mu$ & $\mathrm{kg} /(\mathrm{ms})$ & $21.01 \times 10^{-6}$ & $7.90 \times 10^{-3}$ \\
Surface tension coefficient $\sigma$ & $\mathrm{N} / \mathrm{m}$ & \multicolumn{2}{c}{0.025} \\
Contact angle $\theta$ & 0 & \multicolumn{2}{c}{45}
\end{tabular}

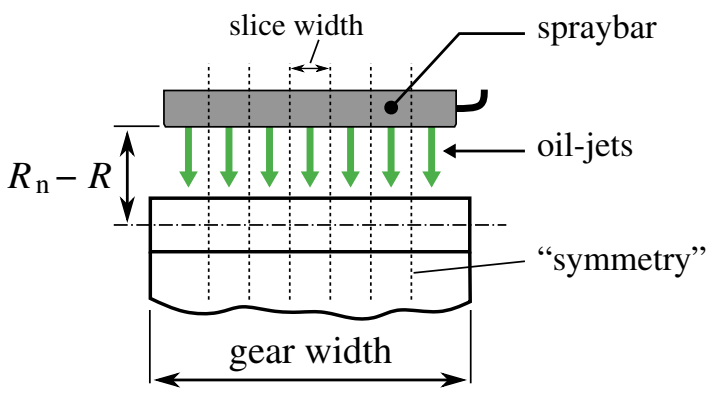

Figure 2. Illustration of the investigated slice of a gear and the nozzle position.

of the simulation data, which allows the extraction of new metrics compared to previous studies. With the new metrics at hand, a comprehensive parameter study is presented, revealing individual effects of the varied operating parameters. In the study the jet velocity, the jet inclination angle, the jet diameter, and the gear speed are varied.

First, the reference geometry, the derived numerical model, and the examined parameter space are introduced. Afterwards, the obtained CFD results and their exploration (postprocessing) are presented. The results of the parameter study are then compared against each other and against analytical approaches where applicable.

\subsection{NUMERICAL SETUP}

\subsection{Geometry and material properties}

The investigated geometry represents a slice of a gear as illustrated in Fig. 2. In practical applications, gears are often cooled by several oil-jets distributed over the width of a gear by spraybars. Thus, the width can be divided into slices with the width of the distance between two jets. Finally, the investigated domain consists of a single gear slice and a single jet nozzle.

The spur gear geometry is further specified by the module of $m=4 \mathrm{~mm}, N=65$ teeth, and the addendum modification coefficient $x_{\mathrm{a}}=0.25$. The nozzle outlet is placed in the radial distance $\left(R_{\mathrm{n}}-R\right)$ of $10 \mathrm{~mm}$ to the gear pitch line and the bore diameter is $d_{\mathrm{n}}=1 \mathrm{~mm}$.

Throughout the isothermal CFD study, the material properties are kept constant. The properties of the considered oil and the surrounding air are taken at a constant pressure of 1 bar and a temperature of $80^{\circ} \mathrm{C}$. The derived material properties are gathered in Table 1. 


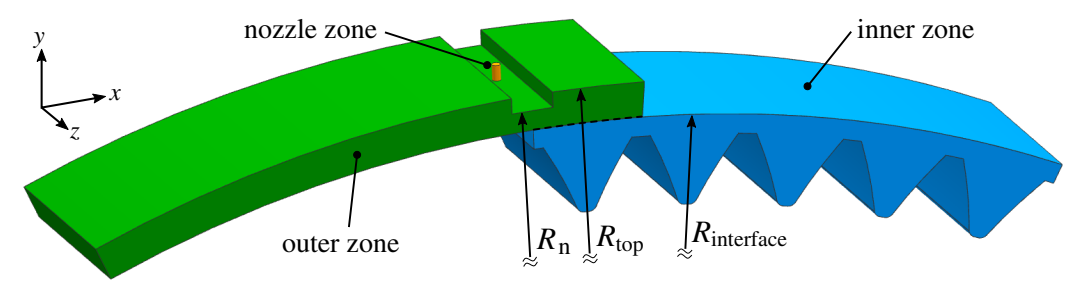

Figure 3. Derived computational domain of the numerical model for $t=0$. The dashed line indicates the overlapping region of the adjacent zones at the sliding interface.

\subsection{CFD model}

\subsubsection{Computational domain}

The geometry presented in the previous section has large geometrical extents compared to the length scales of the oil-jet and the small-scale flow features of the OGI. Instead of modeling the entire gear, a section in the vicinity of the area of interest near the oil-jet impact was extracted. This allows reducing the computational effort compared to a full model assuming a comparable resolution.

As depicted in Fig. 3, a five-teeth segment is extracted from the full gear and the radial confinement is placed at a radius of $R_{\text {top }}=142 \mathrm{~mm}$. The length of the nozzle duct is $l_{\mathrm{n}}=2 \mathrm{~mm}$ and the width of the slice is $w=22.5 \mathrm{~mm}$. The domain consists of three separate zones to enable a modular case assembly and to model the gear motion. For example, a domain with a larger nozzle diameter can, thus, be easily realised by replacing the nozzle zone without modifying the remaining zones. The incorporation of the gear rotation is realized by a socalled Sliding-Interface approach, whereby the interface between the inner and outer zone is used as the sliding plane. The interface is located at $R_{\text {interface }}=137.5 \mathrm{~mm}$.

In Fig. 4, the mesh of the inner zone is shown. The mesh is composed of a quad-dominant surface mesh on the front plane, which is then equidistantly extruded into the axial direction. The surface mesh is generated with an advancing front method, which creates quads in layers orthogonal to walls. Triangles are used at the (inner) regions where the orthogonal fronts converge to enable the transition. The resulting three-streaked seams in the inner region of each tooth gap can be clearly seen. A quad-dominant mesh is chosen, since it is favorable for multiphase simulations with VOF, enabling a more accurate reconstruction of the free surface. Compared to a regular block mesh (all-quad) approach, which was also applied in a pre-study ${ }^{(18)}$, this method is found to yield meshes with fewer cells at a sufficient quality. Previous VOF simulations of liquid jets ${ }^{(19,20)}$ have shown that a mesh resolution of approximately $d_{\text {jet }} / 16$ is sufficient to correctly capture the jet trajectory. Here, the base cell size is set to $d_{\text {jet }} / 4=250 \mu \mathrm{m}$. Through the AMR described in the next section, this criterion is met. Near the gear flanks, the base cell height is reduced to below $50 \mu \mathrm{m}$ to better resolve the boundary layer and the oil film. In combination with the applied AMR, the oil film is found to be properly resolved, since thicknesses below approximately $25 \mu \mathrm{m}$ can be captured reasonably. According to analytical calculations based on the work by DeWinter and Blok ${ }^{(6)}$ thinner film thicknesses $(\approx 10 \mu \mathrm{m})$ are only expected at a certain time interval after the jet impact, which is not in the focus of the current work anymore. While the mesh of the outer zone is generated with a similar approach, the mesh in the cylindrical nozzle zone is block structured. The combined mesh consists of approximately $1.3 \times 10^{6}$ cells in total. 


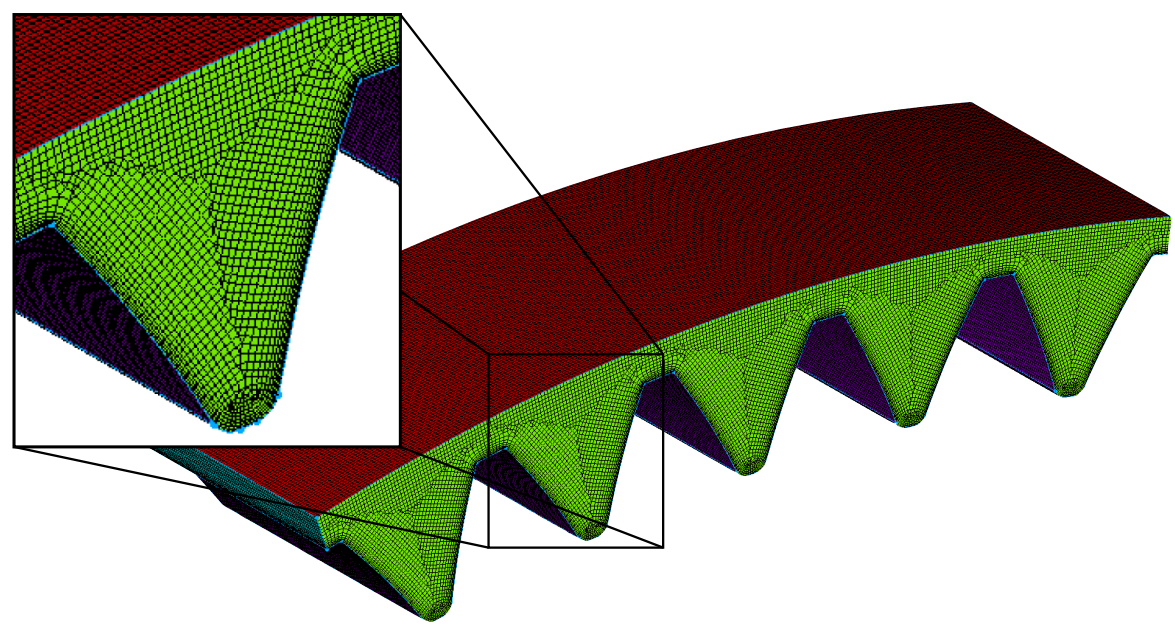

Figure 4. Quad-dominant mesh of the inner computational zone.

\subsubsection{Numerical methodology}

The presented computational domain is employed in conjunction with the VOF method to model the prevailing two-phase flow. The method is based on the work by Hirt and Nichols ${ }^{(21)}$ and extends the underlying Finite Volume method by a phase interface tracking feature. In addition to the well-known governing equations of fluid flows, a further transport equation is included which is used to track the volume fraction of the $\gamma$-th phase $C_{\gamma} \in[0,1]$ assigned to each computational cell. Any fluid property $\phi$ of a cell is then computed by

$$
\phi=\sum_{\gamma} C_{\gamma} \phi_{\gamma}
$$

The actual location of the phase interface is reconstructed from the volume fraction field. In this work, the Compressive scheme is employed. Resolving the sharp free surface requires a fine mesh. To avoid refining the mesh globally a three-leveled AMR and coarsening is applied. Cells with a spatial volume fraction gradient larger than 0.15 get refined up to three times (into up to $8^{3}$ sub-cells). The coarsening is triggered by a gradient of smaller than 0.05 . Surface tension effects are considered by means of a Continuum Surface Force model ${ }^{(22)}$. For turbulence modeling the Unsteady Reynolds-averaged Navier-Stokes model with the $k$ - $\omega$ SST turbulence formulation is selected. Further solver settings involve the PISO scheme for the pressure-velocity coupling and the PRESTO! scheme for the pressure discretisation. Remaining temporal and spatial discretisation schemes are of second order, respectively. For further details on the models, it is referred to the documentation of the employed software ANSYS Fluent release $18.2^{(23)}$.

\subsection{Boundary conditions}

The numerical model is supplemented by a set of boundary conditions (BCs). On all physical walls (gear and nozzle surfaces), a no-slip BC is applied, where hydraulically smooth walls are considered. On the front and back planes of the inner and outer zone, a symmetry BC is used. This reflects the previously described fact that the domain represents an inner slice 
of the spur gear, for which the OGI is assumed to behave symmetrically (c.f. Fig. 2) to its neighboring slices. Modeling the global three-dimensional air flow ${ }^{(4,24)}$ might require to use a domain with larger extents including the side walls of the gears. However, as stated in a previous work ${ }^{(2)}$, aerodynamic effects are not changing the primary oil flow on the flanks. Rotational periodicity is applied in circumferential direction for the respective planes of the inner and outer zone each. On the confinement in radial direction of the outer zone, a pressure outlet is applied. Gravity is omitted, since its effect is negligible ${ }^{(1,2)}$.

The setup contains two mesh interfaces. One is located at the connection between the nozzle and outer zone. The second interface is located between the inner and outer zone. It was already mentioned, that this surface is incorporated to make use of the Sliding-Interface feature. In the current setup this BC is applied in conjunction with the mesh motion of the inner zone and the so-called Periodic-Repeat BC. That is, the inner zone is moving in circumferential direction with a prescribed rotational velocity $\omega$ and slides off along the cylindrically shaped inner interface of the static outer zone. During this process, there occur overlapping and non-overlapping sections as can be seen in Fig. 3. However, the whole interface plane is always a part of the interior fluid domain. While at the overlapping parts the flow variables are directly interpolated between the adjacent cells, an additional step is required at the nonoverlapping parts because no adjacent cells exist. By exploiting the rotational periodicity of the setup, the non-overlapping part of the inner zone is coupled with the non-overlapping part of the outer zone and vice versa. Hence, internally the data is first rotated by one gear segment interval $\left(\frac{2 \pi}{13}\right)$ before the adjacency relations between the neighboring cells for the interpolation are found. To apply this kind of interface, the interface area of the inner and outer zone must be identical. Before the inner zone completely slides off the outer domain, it is rotated back by one segment interval so that there is always a contact between the two zones. During the simulation run, the cell relations at the Sliding-Interface must be updated for each time step. Compared to other strategies to incorporate the gear motion, like Chimera, mesh smoothing, or re-meshing approaches, the computational expenses remain moderate.

Last, at the circular inlet plane of the nozzle zone a uniform velocity profile is employed. The physical simulation time was set to $t_{\mathrm{sim}}=10 \frac{2 \pi}{\omega N}$. Hence, the inner zone passes the outer zone twice in the simulation time. To avoid that the oil-jet further feeds oil to sections of the gear surface, where it already has impinged, the oil volume flow rate at the inlet is set to zero after the physical time $t_{\text {oil-stop }}=5 \frac{2 \pi}{\omega N}$.

\subsection{Parameter variation}

Before the investigated operating conditions are presented, the definitions of quantities used to describe the OGI in this work are introduced. The basic operational parameters are the jet velocity $v_{\text {jet }}$ and the gear speed $\omega$. From latter the circumferential velocity at the pitch circle $v_{\mathrm{p}}=\omega R$ can be derived. In the following context, the velocity ratio

$$
\Sigma=\frac{v_{\text {jet }}}{v_{\mathrm{p}}} \propto \frac{v_{\text {jet }}}{\omega}
$$

will be used as a main dimensionless parameter. A geometric parameter is the inclination angle $\alpha_{\text {jet }}$. Here, the effective inclination angle $\hat{\alpha}_{\text {jet }}$ is introduced, which is defined as the angle between the jet center axis and the normal at its intersection point with the pitch circle (c.f. Fig. 1). Both angles are geometrically related, whereas by the effective angle the nozzle distance to the gear is eliminated. Thus, the effective angle is defined in the interval $\left[-\frac{\pi}{2}, \frac{\pi}{2}\right]$, where an angle of $\pm \frac{\pi}{2}$ corresponds to an oil-jet axis tangential to the pitch circle. The jet 


\section{Table 2}

Varied effective jet inclination angles for two values of $\Sigma$

$\begin{array}{llc}\text { Case } & \boldsymbol{\Sigma}[-] & \hat{\boldsymbol{\alpha}}_{\text {jet }} \text { range }\left[{ }^{\mathbf{0}}\right] \\ \mathrm{A} 1-\mathrm{A} 5 & 0.25 & -23.6 / 0.0 / 11.5 / 23.6 / 36.9 \\ \mathrm{~B} 1-\mathrm{B} 7 & 0.5 & -90.0 /-23.6 / 0.0 / 11.5 / 23.6 / 36.9 / 90.0\end{array}$

diameter is the last geometric parameter to be considered, for which an ideal nozzle with $d_{\mathrm{n}}=d_{\text {jet }}$ is assumed. Thus, the volumetric flow rate $Q$ is simply defined by $Q=\frac{\pi}{4} d_{\text {jet }}^{2} v_{\text {jet }}$. Furthermore, the oil volume fed into one tooth gap is considered by

$$
V_{\mathrm{gap}}=Q \frac{2 \pi}{\omega N}=\frac{\pi^{2}}{2} \frac{d_{\mathrm{jet}}^{2} v_{\mathrm{jet}}}{\omega N} .
$$

Based on a reference case, the derived parameter configurations are presented in Table 2 and 3. The baseline case, in the following indicated by an asterisk $\left(^{*}\right)$, is defined by $\Sigma^{*}=0.5$, $d_{\text {jet }}^{*}=1 \mathrm{~mm}$, and $\hat{\alpha}_{\text {jet }}^{*}=0^{\circ}$ and is equivalent to case B3 in Table 2 . In a first study, the influence of the jet inclination angle is investigated for the two velocity ratios $\Sigma=0.25$ and $\Sigma=0.5$. As given in Table 2, the effective inclination angle was varied in five and seven steps, respectively.

In a second study, the inclination angle is fixed at $\hat{\alpha}^{*}=0^{\circ}$, but the jet velocity and diameter as well as the gear speed are varied as shown in Table 3. Based on the baseline case (B3), the parameters are varied in three steps each. According to the relations in Equations (2) and (3), the varied parameters affect the dependent variables as $\Sigma, Q$, and $V_{\text {gap }}$. To achieve comparable kinematics, the gear speed is adapted (cases II) to retain $\Sigma=0.5$ after the jet velocity was varied (cases I). A similar procedure is adopted for the variation of the jet diameter (cases III to $\mathrm{V}$ ), where additionally the volume flow rates are affected. In total, the conducted parameter study consists of 21 individual simulation cases and, in average, consumed 39910 CPUh per case on four 28-way compute nodes with Intel Xeon E5-2660 v4 processors.

\subsection{RESULTS}

\subsection{Flow phenomenology}

To present the general observable flow phenomenology, the simulation result for the baseline case (B3) is presented first. In Fig. 5, snapshots of three consecutive characteristic flow phases are depicted, where the oil surface with $C_{\text {oil }}=0.5$ is colored by the velocity $v_{\text {rel }}$ relative to the gear rotation. Here and in the following discussions, the third impact of the oil-jet on the dry gear at $t \approx 3 \frac{2 \pi}{\omega N}$ is analysed. As concluded by Fondelli et al. ${ }^{(12)}$, it was found that the preceding impacts contain start-up effects while subsequent impacts yield repetitive results.

There are three main flow phases distinguishable. First, as shown in Fig. 5a, the oil-jet is cut off by the interaction with the preceding gear tooth and penetrates into the subsequent tooth gap. No significant jet disintegration is observable. As described in more detail in a previous work $^{(2)}$, the distance between the nozzle and the gear is too small for major breakup or deflection to occur. Second, the oil-jet impacts on the leading gear flank. As can be seen from Fig. 5b, the impact is associated with a strong interaction between the oil and the gear. 


\section{Table 3}

Varied jet velocities, jet diameters, and gear speeds

Case

Independent parameters

$$
\frac{v_{\text {jet }}}{v_{\text {jet }}^{*}}[-] \quad \frac{d_{\text {jet }}}{d_{\text {jet }}^{*}}[-] \quad \frac{\omega}{\omega^{*}}[-] \quad \frac{Q}{Q^{*}}[-] \quad \frac{V_{\text {gap }}}{V_{\text {gap }}^{*}}[-] \quad \Sigma[-]
$$

Variation of jet velocity $v_{\text {jet }}$

\begin{tabular}{lllllll}
\hline $\mathrm{Ia}(\mathrm{A} 2)$ & $1 / 2$ & 1 & 1 & $1 / 2$ & $1 / 2$ & 0.25 \\
$\mathrm{Ib}$ & $3 / 2$ & 1 & 1 & $3 / 2$ & $3 / 2$ & 0.75
\end{tabular}

Adaption of gear speed $\omega(\Sigma=0.5)$

\begin{tabular}{lllllll}
\hline IIa & $1 / 2$ & 1 & $1 / 2$ & $1 / 2$ & 1 & 0.5 \\
IIb & $3 / 2$ & 1 & $3 / 2$ & $3 / 2$ & 1 & 0.5
\end{tabular}

Variation of jet diameter $d_{\text {jet }}$

\begin{tabular}{lcccccc}
\hline IIIa & 1 & $\sqrt{2}$ & 1 & 2 & 2 & 0.5 \\
IIIb & 1 & $\sqrt{2 / 3}$ & 1 & $2 / 3$ & $2 / 3$ & 0.5
\end{tabular}

Combined variation of $d_{\text {jet }}$ and $v_{\text {jet }}\left(Q, V_{\text {gap }}=\right.$ const. $)$

\begin{tabular}{lllllll}
\hline $\mathrm{IVa}$ & $1 / 2$ & $\sqrt{2}$ & 1 & 1 & 1 & 0.25 \\
$\mathrm{IVb}$ & $3 / 2$ & $\sqrt{2 / 3}$ & 1 & 1 & 1 & 0.75
\end{tabular}

Adaption of gear speed $\omega(\Sigma=0.5)$

\begin{tabular}{ccccccc}
\hline $\mathrm{Va}$ & $1 / 2$ & $\sqrt{2}$ & $1 / 2$ & 1 & 2 & 0.5 \\
$\mathrm{Vb}$ & $3 / 2$ & $\sqrt{2 / 3}$ & $3 / 2$ & 1 & $2 / 3$ & 0.5
\end{tabular}

During the third flow phase, depicted in Fig. 5c, the generated oil film is spreading on the flank and parts of the oil are flung off directly during the impact process, which is then further supported by the acting centrifugal forces. The predicted results agree well with the work by Fondelli et al. ${ }^{(1,12)}$ and Keller et al. ${ }^{(2,13)}$

The illustration of the observable flow phenomenology during the OGI in Fig. 5 shows the complex structure of the prevailing two-phase flow - in its geometric shape and in its temporal evolution. In the subsequent sections established and newly introduced metrics are presented and used to evaluate the conducted CFD study, respectively. The results were found to be representative for cases in which the jet reaches the gear as a compact jet, the impingement depth does not reach values close to 1 and the film width does not exceed the domain width.

\subsection{Impingement and penetration depth}

First, the well-known impingement depth is recalled. As illustrated in Fig. 5b, the impingement depth $D_{\mathrm{i}}$ is defined as the radial distance the jet penetrates into the tooth gap before impinging on the leading flank. Its maximum possible value is the tooth height $H$. In this work, the impingement depth for each simulated case is determined. However, due to the threedimensionality of the jet, the detection of the exact position is not straightforward. Therefore, in addition to the estimated value of $D_{\mathrm{i}}$ which is located at the intersection point of the jet's center axis and the gear flank, also the minimum and maximum values, $D_{\mathrm{i}, \text { min }}$ and $D_{\mathrm{i}, \max }$ as 
(a)

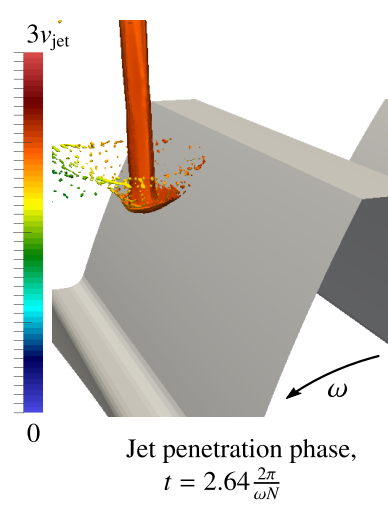

(b)

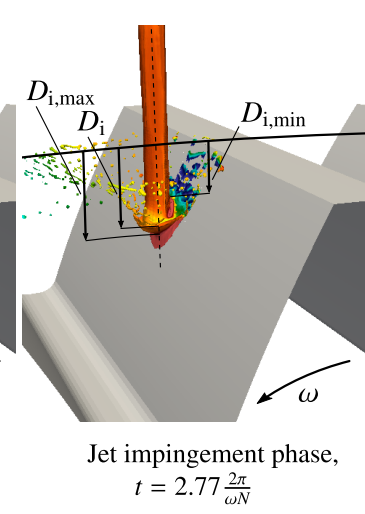

(c)

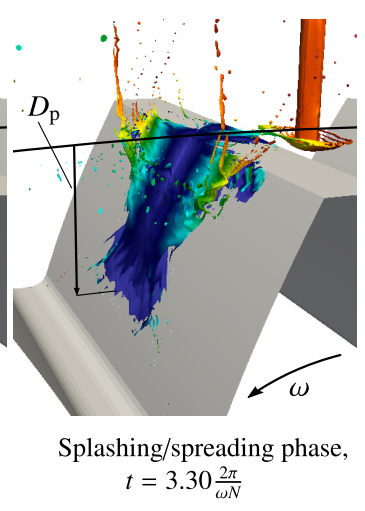

Figure 5. Simulation snapshots of the third jet impingement for the baseline case (B3). The oil surface $\left(C_{\mathrm{oil}}=0.5\right)$ is colored by the relative velocity contour.

depicted in Fig. 5b, are determined from the simulation data. For inclined jets the interval can be considerably large. After the impact, the oil further penetrates towards the bottom land of the gear. This is quantified by the penetration depth $D_{\mathrm{p}}$ as illustrated in Fig. 5c.

The obtained impingement depths for the cases B1-B7 are shown in Fig. 6a. The error bars indicate the maximum and minimum values $D_{\mathrm{i}, \max / \min }$. For moderate positive angles the indicated values for $D_{\mathrm{i}, \max / \min }$ span the widest interval, containing a value of zero as lower bound. In those cases, the jet axis is almost oriented parallel to the flank surface due to the kinematics, leading to a lateral impact of the cylindrical oil-jet (c.f. Fig. 1 right jet). The strong influence of the jet inclination angle is clearly visible. It can be seen, that for large inclination angles the impingement depth tends toward zero. At moderate positive angles, a maximum is observable. This is in good agreement with the analytical solution, which is based on the kinematic model by Akin and Townsend ${ }^{(5)}$. In this model, the impingement depth is analytically determined by considering an infinitesimal thin jet, the well-defined involute profile of the gear teeth, and the imposed kinematics $\left(u_{\text {jet }}, \alpha_{\text {jet }}\right.$ and $\left.\omega\right)$. Note that the jet inclination angle used in the analytical model is defined at the intersection between the jet axis and the gear tip circle, resulting in non-zero values for the effective inclination angles $\hat{\alpha}_{\text {jet }}= \pm 90^{\circ}$, which are defined at the pitch circle in this work.

Compared to the analytical solution, the values of $D_{\text {i }}$ predicted in the CFD simulations are smaller. Akin et al. ${ }^{(4)}$ experimentally observe impingement depths, which are also partly lower compared to the analytical model assuming a given pump pressure. The authors identify the viscous losses in the nozzle and thus the lower jet velocities as the main cause. In the present study this effect can be ruled out, since the jet velocity is directly imposed as a numerical boundary condition. Instead, the simulation results suggest that the interaction of the jet with the gear leads to the decreased impingement depth. The deflection at the top land of the gear teeth and the formation of a disc-shaped oil ligament at the jet tip (c.f. Figs. 5a and 5c) result in a reduction of the effective jet length and thus impingement depth. This cannot be captured in the analytical model, because an infinitesimal thin jet is assumed. Since the deviation between the analytical model and numerically simulated values varies between the investigated cases, further investigation is required to supplement these observations.

The plot additionally contains the predicted maximum penetration depth $D_{\mathrm{p}}$. It can be seen that the penetration depth exceeds the impingement depth by approximately a factor of two. 
(a)

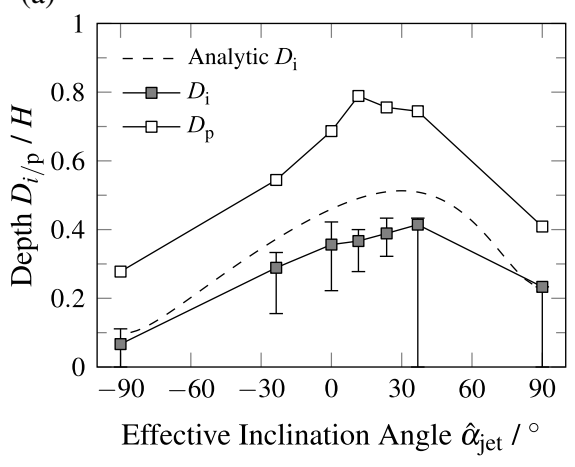

Cases B1-B7 (b)

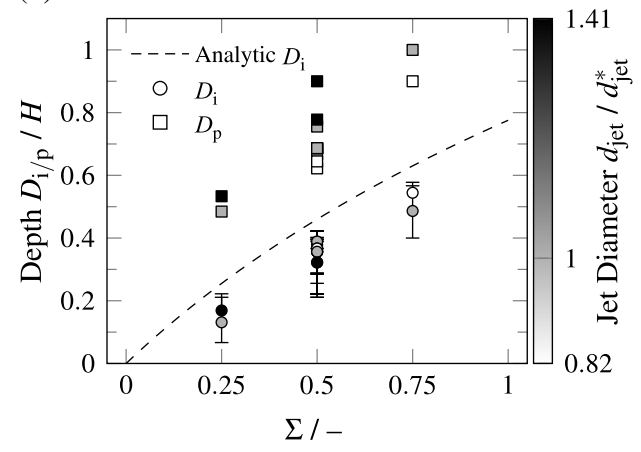

Cases with $\hat{\alpha}_{\text {jet }}=0^{\circ}$

Figure 6. Influence of (a) the effective inclination angle and (b) the jet velocity and diameter on the impingement depth $D_{\mathrm{i}}$ and the maximum penetration depth $D_{\mathrm{p}}$.

Cases with moderate angles show a more pronounced difference between $D_{\mathrm{p}}$ and $D_{\mathrm{i}}$. This effect can be traced back to the higher radial velocity components of the jet in these cases. Since before and after the impact the oil momentum points more strongly towards the gear center, the penetration is enhanced. The described effect of the jet angle on the impingement and penetration depth is observed (not shown) analogously for a reduced jet velocity $(\Sigma=$ $0.25)$ in cases A1-A5.

In Fig. $6 \mathrm{~b}$ the impingement depth and the maximum penetration depth for all radially oriented cases $\left(\hat{\alpha}_{\text {jet }}=0^{\circ}\right)$ are plotted. The jet diameter is represented by the included symbol color. Again, it is observed that the predicted impingement depth is similar to the analytical model by Akin and Townsend ${ }^{(5)}$, whereas slightly smaller values are obtained. The predicted maximum penetration depths exceed the impingement depths in all cases. It can be seen, that at a constant velocity ratio $\Sigma$ larger jet diameters lead to increased penetration depths.

The impingement and penetration depths only give limited information about the spatial oil distribution on the gear flanks and no information about the temporal behavior of the flow. However, through the conducted CFD simulations this information is accessible, which will be discussed in the next section.

\subsection{Temporal evolution of the oil film}

Last, the evolving oil film on the gear flank is tracked by means of the wetted area $A_{\mathrm{w}}$ and the oil film volume $V_{\mathrm{w}}$. For the latter, a connectivity filter is applied to capture the coherent oil structures in contact with the gear flank. Only oil within a wall distance of $1 \mathrm{~mm}$ is considered to omit the volume associated with the cylindrical oil-jet bulk body or ligaments in large distance to the gear flanks as e.g. captured in Figs. 5b and 5c.

The temporal evolution of the wetted area and the connected oil volume on the gear flank for the baseline case (B3) are shown in Fig. 7. After the jet impact, the oil quickly spreads over the flank, leading to a rapid increase of the wetted area until an approximately constant value is reached. In this case, $33 \%$ of the total gear tooth flank area $A_{\mathrm{f}}$ is covered. Although no de-wetting is observable, large amounts of oil are flung off. This can be seen from the trend for the oil volume in contact with the gear flank. After the impact a peak with nearly $100 \%$ is observable, indicating that all oil fed into the tooth gap reaches the flank. However, approximately $80 \%$ of the oil volume is again separated from the tooth within the simulated 


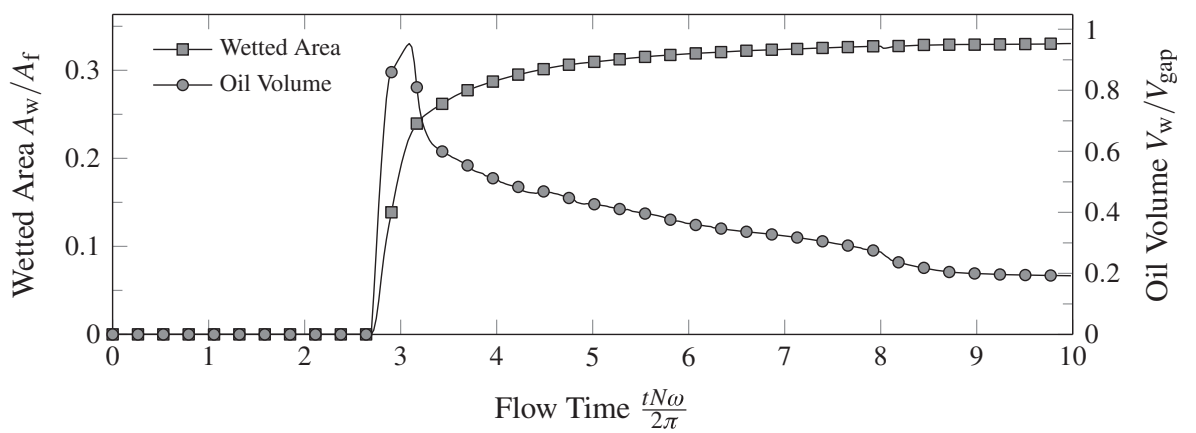

Figure 7. Temporal evolution of the oil film on one gear tooth for the baseline case (B3). Every tenth data point is marked by a symbol.

(a)

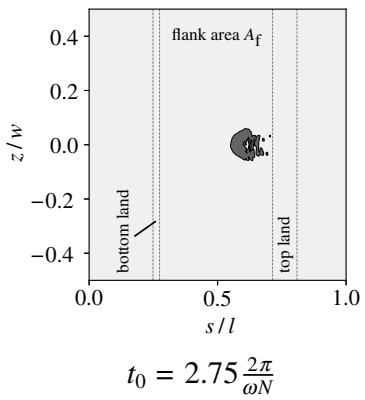

(b)

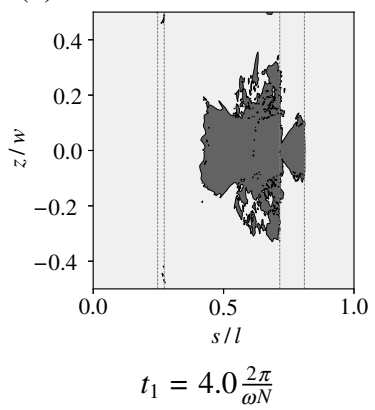

(c)

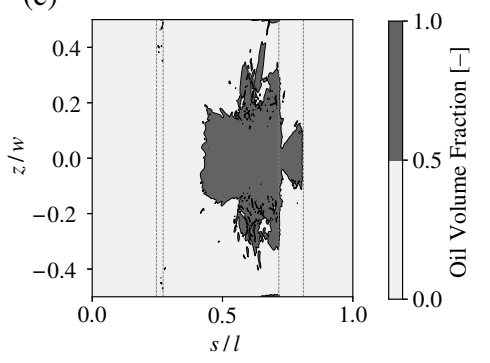

$t_{2}=10.0 \frac{2 \pi}{\omega N}$

Figure 8. Oil distribution maps of one gear tooth for the baseline case (B3) at three time steps.

time span. Comparing this trend with the occurring flow phenomena as seen in Figs. 5a-5c, it is concluded that the steep drop of $V_{\mathrm{w}}$ after the initial peak, is caused by the oil splashing effects. The subsequent decrease with a lower gradient is then caused by the fling-off process driven by centrifugal forces.

In addition, the spatial spreading of the oil on the gear flank is examined. In Fig. 8 maps with the oil distribution on the investigated gear tooth are shown for three time steps. The plots show the volume fraction contour on the two-dimensional plane, spanned by the coordinate axis $z$ in span-wise direction and the contour coordinate $s$ along the gear tooth profile against circumferential direction. The left two dashed lines indicate the lines of sight at the bottom land and the right lines at the top land.

In Fig. 8a the moment of the jet impingement is captured. It can be seen that the initial approximately circular imprint, located at the upper region of the flank, quickly expands. At $t=t_{1}$ the principle shape of the final wetted area has already been reached. In accordance to the previously discussed temporal trend of the total wetted area, the wetted area covers approximately $33 \%$ of the flank (between the line of sights). Afterwards, only small changes in the shape are observable. The same qualitative behavior over time is observable for the 
(a)

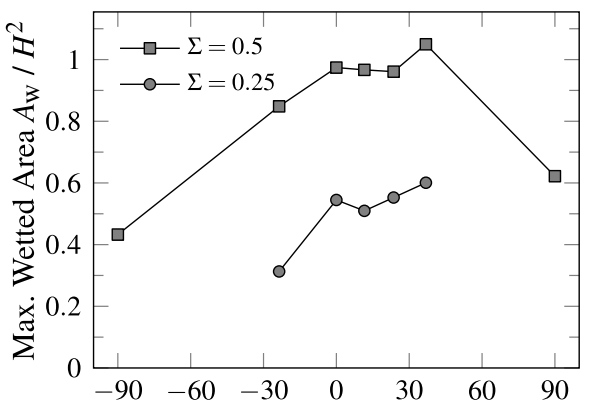

Effective Inclination Angle $\hat{\alpha}_{\text {jet }}{ }^{\circ}$

Cases A1-A5 and B1-B7 (b)

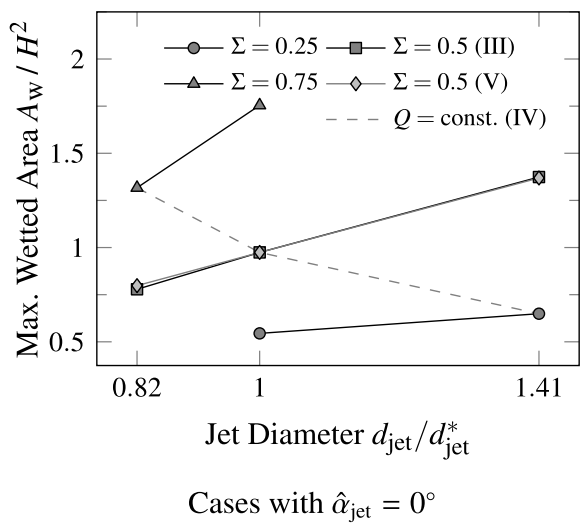

Figure 9. Influence of (a) the effective inclination angle and (b) the jet velocity and diameter on the maximum wetted area $A_{\mathrm{w}}$.

other operating points. In the subsequent sections, only the maximum value of the wetted area is used for comparison.

\subsection{Maximum wetted area}

In this section, the influence of the varied operating parameters on the maximum wetted area is investigated. Note, that during realistic operation of a gearbox, it is not assumed that oil-jets impact and spread on a completely dry gear surface. However, the maximum wetted area is still considered an important indicator for the performance of the cooling and lubricating jet, since it can be understood as an information about areas that are reached by the newly fed coolant.

The influence of the jet inclination angle on the maximum wetted area is presented for cases A1-A5 and B1-B7 in Fig. 9a. Similar to the effect on the impingement depth presented in Fig. $6 \mathrm{a}$, the largest wetted areas are found to occur at moderate positive inclination angles. Large positive and negative angles lead to a significant reduction.

In Fig. 9b the influence of the velocity ratio $\Sigma$ on the maximum wetted area is further investigated in consideration with varied jet diameters and the implications on the oil volume flow rate. From the results for the three different velocity ratios $(\Sigma=0.25,0.5$, and 0.75$)$, a positive effect of the jet diameter on the wetted area can be observed, although the influence of the parameter $\Sigma$ is more dominant. It is also observed, that for cases III and V the same wetted areas are predicted, even though the cases operate at different absolute jet velocities and gear speeds. This is an indication that the initial spreading is rather dominated by inertia effects than by centrifugal forces. This is again in accordance with the observations from Figs. $8 \mathrm{a}-8 \mathrm{c}$, where it was concluded that during the fling-off process the shape of the wetted area does not significantly change. If a constant oil feed volume flow rate is considered, the effect of the jet diameter reverses. For cases IV, the volume flow rate is kept constant, leading to reduced velocity ratios $\Sigma$ along the dashed line. Hence, if a large wetted area is to be achieved, a small oil-jet is beneficial. Note, that this also requires higher pump pressures, which has to be taken into account for technical applications. 


\subsection{CONCLUSION}

In this work, a numerical setup to investigate the flow phenomena associated with the OGI by means of the VOF method is presented. The numerical model consists of a periodic five-teeth segment of a spur gear and the gear motion is incorporated by a Sliding-Interface approach. The setup is applied in a parameter study consisting of 21 cases to investigate the effect of the jet velocity, the jet diameter, the jet inclination angle, and the gear speed.

To examine the individual influences the well-known impingement depth was elaborated. Additional metrics were defined to exploit the three-dimensional and transient data available through the CFD simulations, that are:

- the minimum and maximum impingement depth $D_{\mathrm{i}, \max / \mathrm{min}}$,

- the maximum penetration depth $D_{\mathrm{p}}$,

- the temporal and spatial evolution of the wetted area $A_{\mathrm{w}}$ of the gear tooth, and

- the temporal evolution of the oil volume $V_{\mathrm{w}}$ in contact with the gear tooth.

In accordance to an analytical model, the strong influence of the ratio of jet velocity to gear pitch line velocity $\Sigma$ and the jet inclination angle $\hat{\alpha}_{\text {jet }}$ on the impingement depth is reproduced. Compared to the analytical model, slightly smaller impingement depths were predicted. The penetration depth was found to exceed the impingement depth for all cases, while the difference is amplified with larger jet diameters.

The temporal evolution of the oil film is presented for the first time by means of the wetted area and the connected oil volume. The respective trends show that the oil spreading on the gear flanks occurs quickly after the jet impact. While parts of the oil immediately loose contact to the gear tooth due to splashing effects, other parts are flung off by centrifugal forces over a longer time interval. In addition to the temporal analysis, the presented oil distribution maps yield further insights into the spatial distribution of the oil on the gear flanks.

The influence of the jet inclination angle on the maximum wetted area is similar to its influence on the impingement depth. Largest wetted areas are achieved at moderate positive angles. The velocity ratio $\Sigma$ was found to be the dominant parameter. While increased jet diameters lead to larger wetted areas, thinner jets appear to be beneficial if a constant oil volume flow rate per jet is considered.

While a validation of this work with experimental data is pending, the detail of the numerically obtained results, the newly presented metrics, and the observed effects the work contributes to the further understanding of the OGI and its implication on the oil-jet lubrication and cooling system of gearboxes in civil aero engines. In future work, the effect of further parameters like the material properties (oil viscosity), gear geometry, and nozzle arrangement could be studied. Furthermore, the investigation of the OGI for interlocking gear pairs, gears embedded in more realistic gearbox environments, or the OGI including heat transfer effects is desirable. Latter may be evaluated with respect to the presented wetted areas.

\section{ACKNOWLEDGEMENTS}

The authors acknowledge support by the state of Baden-Württemberg through bwHPC. The research leading to these results has received funding from the BMWi ("Bundesministerium für Wirtschaft und Energie") as part of the research program LuFo V ("Luftfahrtforschungsprogramm"). The authors gratefully acknowledge Rolls-Royce Deutschland Ltd \& Co KG for the outstanding collaboration. 


\section{REFERENCES}

1. Fondelli, T., Andreini, A., Da Soghe, R., Facchini, B. and Cipolla, L. Numerical simulation of oil jet lubrication for high speed gears, International Journal of Aerospace Engineering, 2015, 2015, id:752457. doi:10.1155/2015/752457.

2. Keller, M.C., Braun, S., Wieth, L., Chaussonnet, G., Dauch, T.F., Koch, R., Schwitzke, C. and BAUER, H.J. Smoothed particle hydrodynamics simulation of oil-jet gear interaction, Journal of Tribology, 2019, 141, (7), id:071703. doi:10.1115/1.4043640.

3. McCAIn, J.W. and AlsANDOR, E. Analytical aspects of gear lubrication on the disengaging side, A S L E Transactions, 1966, 9, (2), pp 202-211. doi:10.1080/05698196608972136.

4. AkIN, L.S., Mross, J.J. and Townsend, D.P. Study of lubricant jet flow phenomena in spur gears, Journal of Lubrication Technology, 1975, 97, (2), pp 283-288. doi:10.1115/1.3452576.

5. AKIN, L.S. and TownsEnd, D.P. Lubricant jet flow phenomena in spur and helical gears with modified addendums-for radially directed individual jets, 5th International Power Transmission and Gearing Conference, vol. 1, Chicago, IL, USA, pp 367-374.

6. DeWInter, A. and BloK, H. Fling-off cooling of gear teeth, Journal of Engineering for Industry, 1974, 96, (1), pp 60-70. doi:10.1115/1.3438331.

7. Kromer, C., Cordes, L., Keller, M.C., Schwitzke, C. and Bauer, H.J. Analytical solution to the heat transfer in fling-off cooling of spur gears, Journal of Heat Transfer, 2019, 141, (8), id:082103. doi:10.1115/1.4043894.

8. AKIN, L.S. An interdisciplinary lubrication theory for gears (with particular emphasis on the scuffing mode of failure), Journal of Engineering for Industry, 1973, 95, (4), pp 1178-1195. doi: $10.1115 / 1.3438267$.

9. Schober, H. Untersuchungen zur Einspritzschmierung der Stirnradgetriebe, PhD Thesis, Institut für Maschinenkonstruktion und Getriebebau, University of Stuttgart, Stuttgart, Germany, 1983.

10. Massini, D., Fondelli, T., Facchini, B., Tarchi, L. and Leonardi, F. Experimental investigation on power losses due to oil jet lubrication in high speed gearing systems, Proceedings of ASME Turbo Expo 2017: Turbomachinery Technical Conference and Exposition, vol. 5B: Heat Transfer, Charlotte, NC, USA, id:V05BT15A030. doi:10.1115/gt2017-64703.

11. YAZDANI, M. and Soteriou, M.C. A novel approach for modeling the multiscale thermo-fluids of geared systems, International Journal of Heat and Mass Transfer, 2014, 72, pp 517-530. doi:10.1016/j.ijheatmasstransfer.2014.01.035.

12. Fondelli, T., Andreini, A., Da Soghe, R., Facchini, B. and Cipolla, L. Volume of fluid (VOF) analysis of oil-jet lubrication for high-speed spur gears using an adaptive meshing approach, Proceedings of ASME Turbo Expo 2015: Turbine Technical Conference and Exposition, vol. 7A: Structures and Dynamics, Montreal, Canada, id:V07AT31A006. doi:10.1115/GT2015-42461.

13. Keller, M.C., Braun, S., Wieth, L., Chaussonnet, G., Dauch, T.F., Koch, R., Schwitzke, C. and BAUER, H.J. Smoothed particle hydrodynamics simulation of oil-jet gear interaction, Proceedings of ASME Turbo Expo 2017: Turbomachinery Technical Conference and Exposition, vol. 2B: Turbomachinery, Charlotte, NC, USA, id:V02BT41A019. doi:10.1115/GT2017-63594.

14. Ambrose, S., Morvan, H. and Simmons, K. Investigation of oil jet impingement on a rotating gear using lattice boltzman method (LBM), Proceedings of ASME Turbo Expo 2018: Turbomachinery Technical Conference and Exposition, vol. 1: Aircraft Engine; Fans and Blowers; Marine, Oslo, Norway, id:V001T01A028. doi:10.1115/gt2018-76371.

15. Arisawa, H., Shinoda, Y., Tanaka, M., Goi, T., Akahori, H. and Yoshitomi, M. Classification of fluid dynamic loss in aeroengine transmission gears: Experimental analysis and CFD validation, Proceedings of ASME Turbo Expo 2017: Turbomachinery Technical Conference and Exposition, vol. 1: Aircraft Engine; Fans and Blowers; Marine; Honors and Awards, Charlotte, NC, USA, id:V001T01A004. doi:10.1115/gt2017-63208.

16. Arisawa, H., Shinoda, Y., Tanaka, M., Goi, T., Akahori, H. and Yoshitomi, M. Classification and modeling of fluid dynamic loss in aeroengine transmission gears, Journal of Engineering for Gas Turbines and Power, 2019, 141, (6), id:061012. doi:10.1115/1.4042509.

17. Liu, H., Link, F., Lohner, T. and Stahl, K. Computational fluid dynamics simulation of geared transmissions with injection lubrication, Proceedings of the Institution of Mechanical Engineers, Part C: Journal of Mechanical Engineering Science, 2019, 233, (21-22), pp 7412-7422. doi:10.1177/0954406219865920. 
18. Keller, M.C., Braun, S., Wieth, L., Chaussonnet, G., Dauch, T., Koch, R., HöFler, C. and BAUER, H.J. Numerical modeling of oil-jet lubrication for spur gears using smoothed particle hydrodynamics, Proceedings of the 11th International SPHERIC Workshop, Munich, Germany, pp 69-76. doi:10.5445/IR/1000063565.

19. Herrmann, M. Detailed numerical simulations of the primary atomization of a turbulent liquid jet in crossflow, Journal of Engineering for Gas Turbines and Power, 2010, 132, (6), id:061506. doi:10.1115/1.4000148.

20. KRUG, M. Untersuchung der Ölversorgung in einer triebwerkstypischen Lagerkammer mittels experimenteller und numerischer Methoden, Dissertation, Institut für Thermische Strömungsmaschinen, Karlsruhe Institute of Technology, Karlsruhe, Germany, 2019.

21. Hirt, C. and Nichols, B. Volume of fluid (VOF) method for the dynamics of free boundaries, Journal of Computational Physics, 1981, 39, (1), pp 201-225. doi:10.1016/0021-9991(81)90145-5.

22. Brackbill, J.U., Kothe, D.B. and Zемach, C. A continuum method for modeling surface tension, Journal of Computational Physics, 1992, 100, (2), pp 335-354. doi:10.1016/0021-9991(92)90240-y.

23. ANSYS, Inc. ANSYS ${ }^{\circledR}$ Fluent, Release 18.2, Fluent User's Guide, 2017.

24. Massini, D., Fondelli, T., Andreini, A., Facchini, B., Tarchi, L. and Leonardi, F. Experimental and numerical investigation on windage power losses in high speed gears, Proceedings of ASME Turbo Expo 2017: Turbomachinery Technical Conference and Exposition, vol. 5B: Heat Transfer, Charlotte, NC, USA, id:V05BT15A034. doi:10.1115/gt2017-64948. 